\title{
Aggressive intraosseous lipoma of the intermediate phalanges of the thumb
}

\author{
KAZUHIKO HASHIMOTO, SHUNJI NISHIMURA, RYOSUKE KAKINOKI and MASAO AKAGI \\ Department of Orthopedic Surgery, Kindai University Hospital, Osaka-Sayama, Osaka 589-8511, Japan
}

Received January 30, 2018; Accepted May 15, 2018

DOI: $10.3892 / \mathrm{mco} .2018 .1631$

\begin{abstract}
Intraosseous lipomas occurring in the bones of the upper limbs are very rare. The tumor often occurs in long bones, especially the calcaneus. Usually patients with intraosseous lipomas present with mild clinical features. Thus far, bone destruction caused by the tumor has not been reported. The present study reported a case of an aggressive intraosseous lipoma that developed in the intermediate phalanges of the thumb. This is an extremely rare case with extraosseous development, which occurred at a rare site. A 47-year old woman presented to us with right thumb pain and swelling. Computed tomography and magnetic resonance imaging revealed a mass extending to the outer edge of the phalangeal bone. The patient was treated with surgery the remove the tumor. Artificial bone was used to refill the area due to the lack of cancellous bone. During the clinical management of lipomas, it is important to consider that intraosseous lipomas may spread out of the bone; moreover, the tumor should be removed immediately to help reduce the possibility of bone destruction.
\end{abstract}

\section{Introduction}

An intraosseous calcaneal lipoma was first described in 1976 (1). These are benign bone tumors that are rather uncommon, despite the abundance of adipose connective tissue in the bone marrow (1). One known site that intraosseous lipomas may occur is within the calcaneus bone. Patients with intraosseous lipomas are often asymptomatic, and several cases are incidentally discovered (2). We herein

Correspondence to: Dr Kazuhiko Hashimoto, Department of Orthopedic Surgery, Kindai University Hospital, 377-2 Ohno-Higashi, Osaka-Sayama, Osaka 589-8511, Japan

E-mail: hazzhiko@med.kindai.ac.jp

Abbreviations: CDK4, cyclin-dependent kinase 4; CT, computed tomography; MDM2, murine double minute 2; MRI, magnetic resonance imaging; STIR, short TI inversion recovery

Key words: intraosseous lipoma, intermediate phalanges, thumb, bone destruction, symptomatic report the very rare case of intraosseous lipoma of thumb with bone destruction.

\section{Case report}

This is a case of a 47-year old woman who presented with right thumb pain and swelling (Fig. 1A and B). Her past medical history did not include any trauma or any other causative clinical condition. She also stated that there was no family history of any type of cancer. There was no limitation in the range of movement. A radiographic image showed a soap-bubble appearance, indicating bone destruction and rupture of the bone cortex of the intermediate phalanges of the thumb (Fig. 1C and D). A computed tomography (CT) image confirmed the presence of a tumor with fat concentration, bone destruction in the intermediate phalanges, and rupture of the bone cortex (Fig. 1E and F). Magnetic resonance imaging $(\mathrm{MRI})$ revealed a low-intensity mass extending to the outer area of the phalangeal bone with inner mosaic intensity in a T1-weighted image (Fig. 2A). A T2-weighted image also showed a high-intensity mass with inner mosaic intensity (Fig. 2B), while a short TI inversion recovery (STIR) image showed a low-intensity mass (Fig. 2C). The tumor was resect by making a zigzag skin incision from the palm side. A further incision was made from the A 0 pulley to the A 2 pulley, and the tumor was identified by moving the flexor tendon to the side (Fig. 2D). The tumor was found adhered to the bone wall, and had expanded by destroying several bone walls. To remove the tumor, we scraped the bone wall where adhesion of the tumor was evident. The tumor had also adhered to the surrounding soft tissue. After washing, beta-tricalcium phosphate was used to fill in the bone defect (Fig. 2E and F). Sutures were placed extending from the A0 pulley to the A2 pulley. A Penrose drain was placed subcutaneously, and the subcutaneous tissue and skin were sutured. The resected tumor showed a lipomatous image profile and was elastic in texture. Hematoxylin-eosin staining of the tumor section showed both necrotic fat tissue and fibrous tissues (Fig. 3A). The immunohistochemical staining was negative for murine double minute 2 (MDM2) and cyclin-dependent kinase 4 (CDK4) (Fig. 3B and C). No obvious abnormal findings were found in postoperative blood test results (Table I). Currently, 6 months after surgery, tumor recurrence or any thumb malfunction has not been observed. 

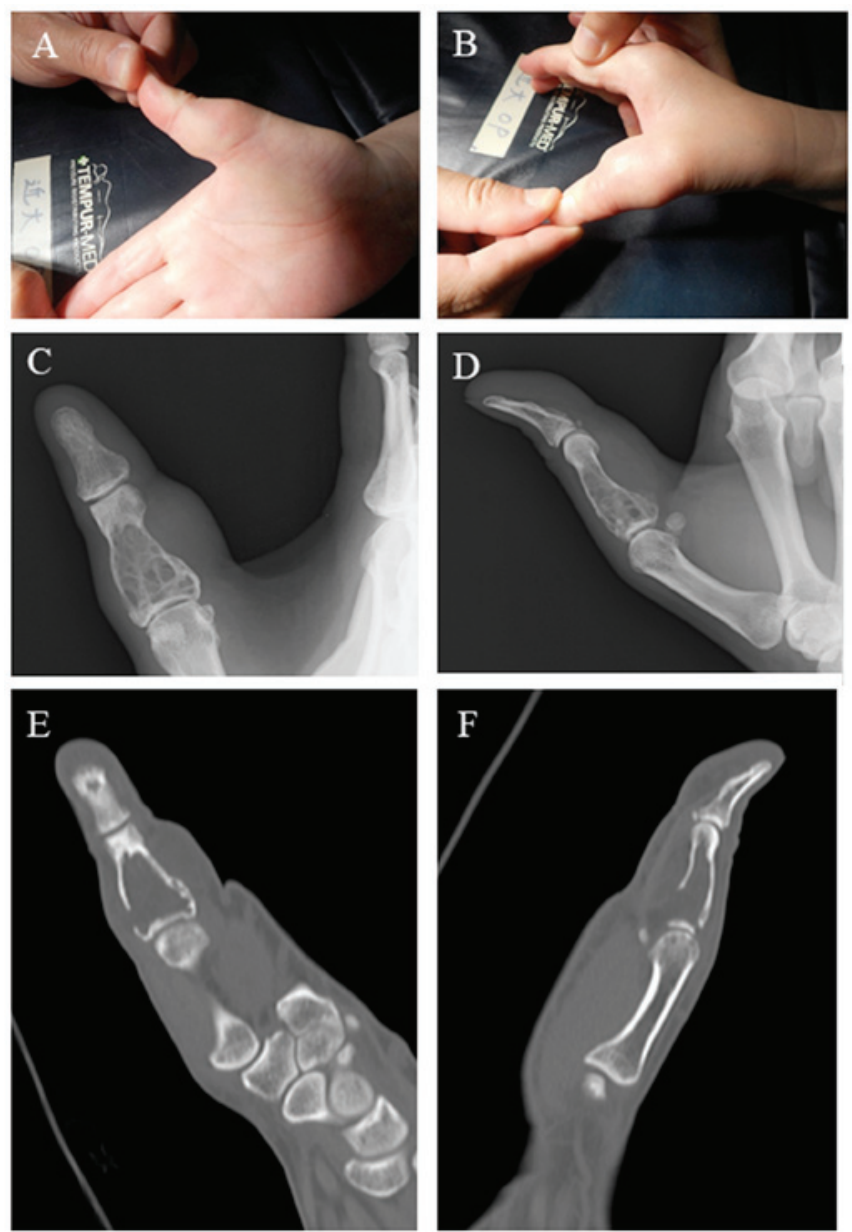

Figure 1. Exterior appearance, radiographic and computed tomography scans Exterior appearance of the thumb (A and B). Radiographic scans (C and D) Computed tomography (CT) scans (E and F).

\section{Discussion}

Intraosseous lipomas are a rare type of tumor. Furthermore, tumors developing in the upper limbs are very rare (7\%) (3). Only one case of intraosseous lipoma in the hand has been reported previously (4). To the best of our knowledge, this is the first reported case of an intraosseous lipoma occurring from the intermediate phalanges of the thumb. Approximately $70 \%$ of patients with intraosseous lipomas present with pain (5). Pathological fractures are a rare cause of pain in intraooseous lipomas (3). The cause of pain in this case was thought to be due to bone destruction. An intraosseous lipoma with multiple region involvement is also quite rare (6). As in the present case, cases of single lesions account for the majority of intraosseous lipomas. Discrete lipomas should be distinguished from multiple lipomatosis (7), in which the fat deposition may be due to associated endocrine abnormalities such as type IV hyperlipidemia (8) or other conditions such as macrodystrophia lipomatosa (3). The identification of fat density on CT is usually considered diagnostic of an intraosseous lipoma (9), although other lesions such as osteomyelitis may demonstrate low attenuation values due to the presence of fat-laden histiocytes (10). The fat component of the intraosseous lipoma is easily recognized on MRI scans by high signal intensity on both T1-weighted and T2-weighted scans, and fat suppression on STIR or other fat suppression sequences.
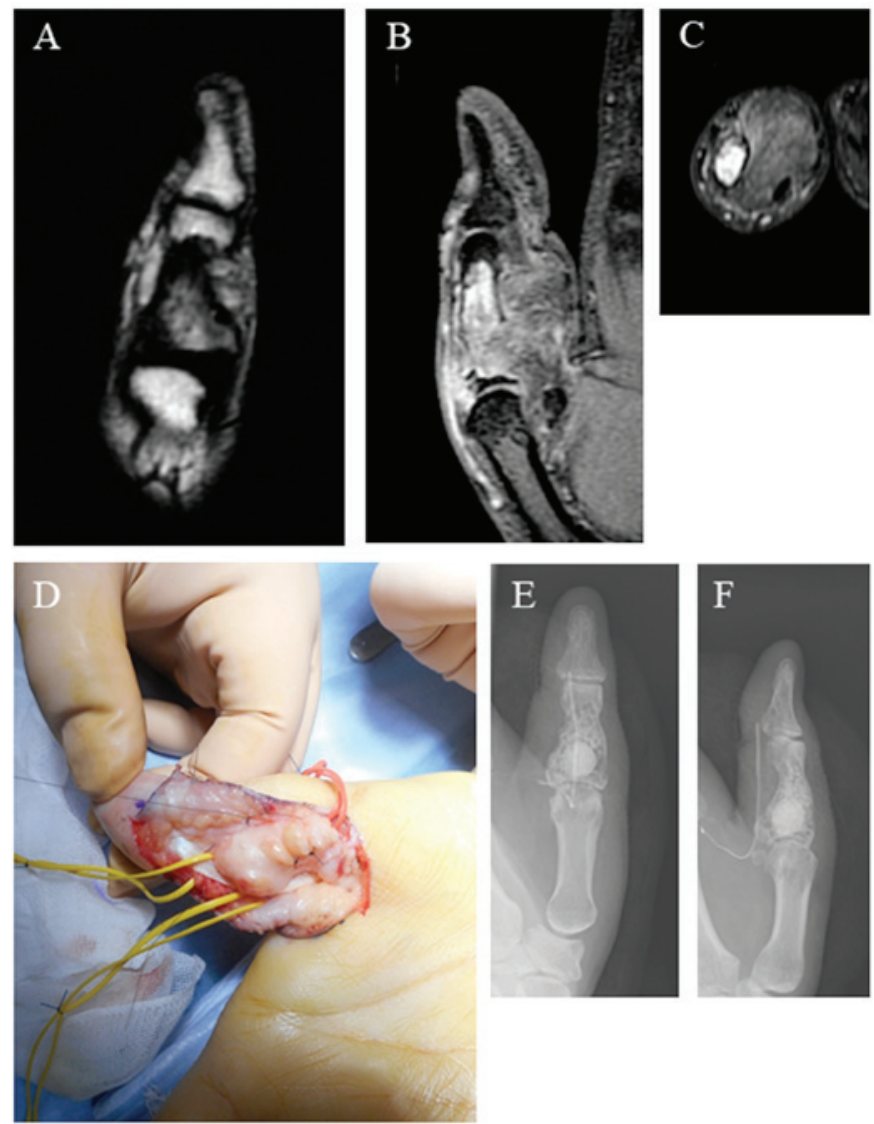

Figure 2. Magnetic resonance imaging scan and surgical findings. T1-weighted magnetic resonance imaging (MRI) scan (A). T2-weighted MRI scan (B). Short TI inversion recovery (STIR) MRI scan (C). Surgical findings (D). Radiographic scans after surgical treatment (E and F).

Cysts are commonly seen on MRI scans and have well-demarcated borders (6). Signal intensity is intermediate on T1-weighted sequences and very high on T2-weighted and fat-suppressed scans (11). The appearances of lipomas on radiography, CT, and MRI correspond to the pathological staging system (2). Milgram states that stage 1 lesions are purely radiolucent with resorption of pre-existing bone and expansion or remodeling in 50\% of all cases (2). In stage 2 lesions, localized areas of calcification may be seen and are typically central, but may also be peripheral. At stage 3 , reactive ossification is prominent around the calcified fat in the outer rim of the lesion. Peripheral or central calcification fills much of the lesion, but expansion is present in some cases (2). Clinical imaging indicated stage 2 disease in the current case. Bone expansion has been reported to be typically minimal or absent, although prominent expansion has been described in the fibula (12), spine and sacrum (13), and skull (14). One previous study reported that smaller lesions in the long bones may lead to bone expansion or focal infarction or may destroy the cortical bone (15). Interestingly, in the current case, destruction of the bone wall was observed in addition to bone expansion. Milgram also proposed three stages based on the histological appearances of intraosseous lipomas (2). Stage 1 lesions are defined as containing viable mature lipocytes interspersed with fine bony trabeculae. The fat is identical to subcutaneous fat on chromatography. There is no cellular atypia, mitosis, or capsular tissue. Stage 2 lesions develop areas of infarction due to expansion of fat cells within rigid trabeculae (2). The adipose tissue 
Table I. Blood test results.

\begin{tabular}{lccc}
\hline Test component & One month before presentation & Time of presentation & One day after surgery \\
\hline CRP level $(\mathrm{mg} / \mathrm{dl})$ & 0.014 & 0.022 & 0.321 \\
eGFR & 90 & 95 & 93 \\
AST level $(\mathrm{U} / \mathrm{l})$ & 17 & 19 & 15 \\
ALT level $(\mathrm{U} / \mathrm{l})$ & 17 & 18 & 15 \\
WBC count $\left(\mathrm{x} 10^{3} \mu \mathrm{l}\right)$ & 6.27 & 5.75 & 7.68 \\
HGB count $(\mathrm{g} / \mathrm{dl})$ & 13.7 & 12.1 & 12.4 \\
PLT count $\left(\mathrm{x} 10^{4} \mu \mathrm{l}\right)$ & 28.5 & 23.5 & 24.5 \\
\hline
\end{tabular}

CRP, C-reactive protein; eGFR, estimated glomerular filtration rate; AST, aspartate aminotransferase; ALT, alanine aminotransferase; WBC, white blood cell; HGB, hemoglobin; PLT, platelet.
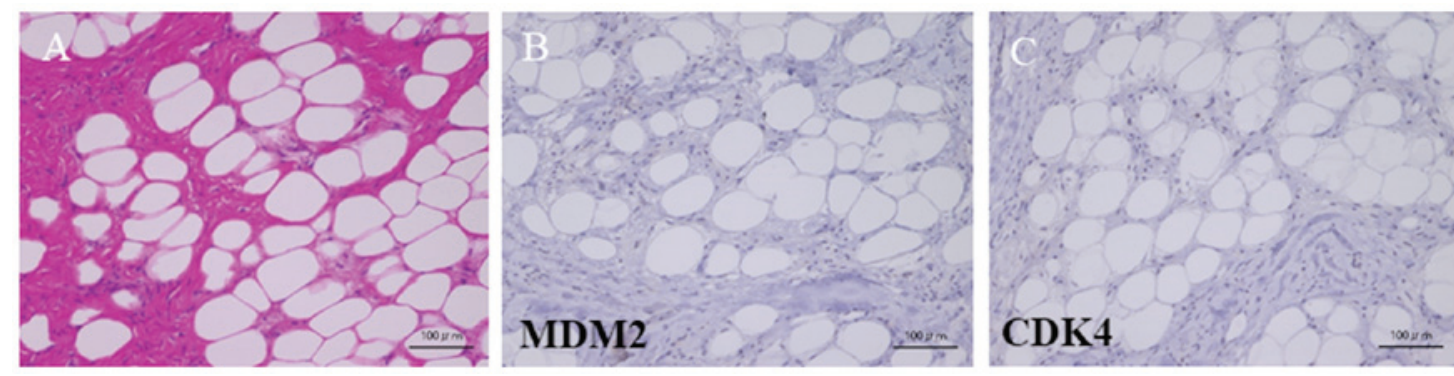

Figure 3. Hematoxylin-eosin-stained image and immunohistochemical findings. Hematoxylin-eosin-stained image of a tumor section (A). Immunohistochemical findings (B and C). Murine double minute 2 (MDM2) staining (B). Cyclin-dependent kinase 4 (CDK4) staining (C). Scale bar, $100 \mu \mathrm{m}$.

is partly necrotic with loss of nuclei, and foamy macrophages may be present. Some portions of necrotic fat become calcified. Reactive ossification may develop adjacent to areas of necrotic fat, and resembles primitive woven bone. Extension of infarction to involve the whole lesion leads to a stage 3 lipoma, with necrotic fat, calcified fat, cyst formation, and reactive peripheral or central new bone formation, although these features may be seen to a lesser extent in the stage 2 lesions. In this respect, lipomas resemble bone infarcts, although the bony trabeculae are resorbed in stage 3 lipomas, and are considered to be a distinguishing feature (2). Infarcts also typically have peripheral calcification. Cysts have also been described in lesions other than lipomas, such as bone infarcts and fibrous dysplasia. In this case, histological findings resembled stage 2 of Milgram's classification.

The interesting feature of the current case, in the context of stage 2 of Milgram's classification, is that fibrosis was observed. Most lipomas can be managed conservatively. The primary indications for surgical intervention are suspicion or evidence of malignancy (although rare) (16), or the risk of pathological fracture (although there are no reports of this complication) (17). Other indications include cosmetic deformity or pain. Surgical treatment usually consists of curettage and packing with bone chips, similar to the treatment offered in the current case. The limitation of the current study is that we were not able to show a correlation between blood test findings and the disease condition. Further research is needed to help identify markers that can be used to help recognize the disease in patients.

In conclusion, we report a case of a rare intraosseous lipoma occurring from the intermediate phalanges of the thumb. Surgical resection was successful. Bone tumors that occur in the intermediate phalanges of the thumb should be screened for intraosseous lipomas and treatment should be decided accordingly. It should also be noted that even benign tumors may cause bone destruction.

\section{Acknowledgements}

Not applicable.

\section{Funding}

No funding was received.

\section{Availability of data and material}

The authors declare that data and material can be made available on request.

\section{Authors' contributions}

$\mathrm{KH}, \mathrm{SN}$ and $\mathrm{RK}$ were involved in the acquisition of data. $\mathrm{KH}$ and $\mathrm{SN}$ analyzed the data. $\mathrm{KH}$ and MA prepared the manuscript.

\section{Ethics approval and consent to participate}

The Ethics Committee of the Kindai University Faculty of Medicine approved the present study and patients provided informed consent to participate. 


\section{Consent for publication}

The patient provided written informed consent.

\section{Competing interests}

The authors declare that they have no competing interests.

\section{References}

1. Poussa $\mathrm{M}$ and Holmström T: Intraosseous lipoma of the calcaneus. Report of a case and a short review of the literature. Acta Orthop Scand 47: 570-574, 1976

2. Milgram JW: Intraosseous lipomas. A clinicopathologic study of 66 cases. Clin Orthop Relat Res: 277-302, 1988

3. Campbell RS, Grainger AJ, Mangham DC, Beggs I, Teh J and Davies AM: Intraosseous lipoma: Report of 35 new cases and a review of the literature. Skeletal Radiol 32: 209-222, 2003.

4. Bower G, Hosny S and Umarji SI: Intraosseous lipoma of the scaphoid. J Hand Surg Eur Vol 37: 799-800, 2012.

5. Levin MF, Vellet AD, Munk PL and McLean CA: Intraosseous lipoma of the distal femur: MRI appearance. Skeletal Radiol 25: 82-84, 1996.

6. Rosenblatt EM, Mollin J and Abdelwahab IF: Bilateral calcaneal intraosseous lipomas: A case report. Mt Sinai J Med 57: 174-176, 1990.
7. Rehani B and Wissman R: Multiple intraosseous lipomatosis: A case report. Cases J 2: 7399, 2009.

8. Freiberg RA, Air GW, Glueck CJ, Shikawa T and Abrams NR: Multiple intraosseous lipomas with type-IV hyperlipoproteinemia. A case report. J Bone Joint Surg Am 56: 1729-1732, 1974.

9. Ketyer S, Brownstein S and Cholankeril J: CT diagnosis of intraosseous lipoma of the calcaneus. J Comput Assist Tomogr 7: 546-547, 1983.

10. Ramos A, Castello J, Sartoris DJ, Greenway GD, Resnick D and Haghighi P: Osseous lipoma: CT appearance. Radiology 157: 615-619, 1985.

11. Propeck T, Bullard MA, Lin J, Doi K and Marte W: Radiologic-pathologic correlation of intraosseous lipomas. AJR Am J Roentgenol 175: 673-678, 2000.

12. Gero MJ and Kahn LB: Case report 498: Intraosseous lipoma of the distal end of the fibula with focal infarction. Skeletal Radiol 17: 443-446, 1988.

13. Hanelin LG, Sclamberg EL and Bardsley JL: Intraosseous lipoma of the coccyx. Report of a case. Radiology 114: 343-344, 1975.

14. Yasuda Y, Tsukada S, Okada T and Haseda Y: Intraosseous lipoma of the skull: A report of two cases. Ann Plast Surg 18: 74-80, 1987.

15. Leeson MC, Kay D and Smith BS: Intraosseous lipoma. Clin Orthop Relat Res: 186-190, 1983.

16. Milgram JW: Malignant transformation in bone lipomas. Skeletal Radiol 19: 347-352, 1990.

17. Richardson AA, Erdmann BB, Beier-Hanratty S, Lautz D, Jacobs PM, Julsrud ME and Ringstrom JB: Magnetic resonance imagery of a calcaneal lipoma. J Am Podiatr Med Assoc 85: 493-496, 1995. 